\title{
The Impact of Using Non-Birth Sex on the Interpretation of Spirometry Data in Subjects With Air-Flow Obstruction
}

\author{
Jeffrey M Haynes RRT RPFT FAARC and Ralph W Stumbo RRT CPFT
}

\begin{abstract}
BACKGROUND: Sex is an important determinant of lung capacity and function. This study examined the impact of using non-birth sex on the interpretation of spirometry data in transgender subjects with air-flow obstruction. METHODS: This study was a retrospective analysis of anonymous spirometry data. Eighty adult male and 80 adult female subjects were chosen from the database via random sampling. FVC, $\mathrm{FEV}_{1}$, and $\mathrm{FEV} / \mathrm{FVC}$ were collected and analyzed. Differences in percent of predicted, $Z$ scores, classification of disease severity, and the incidence of a value migrating above or below the lower limit of normal between sex assignments were examined. RESULTS: For born male subjects, percent of predicted for FVC and FEV 1 were significantly higher when the female sex was used: $100.5 \%$ versus $118.5 \%$ and $78 \%$ versus $91.5 \%$, respectively $(P<.001)$. FEV $1 / F V C Z$ score was -2.53 for male sex and -2.65 for female sex $(P=.004)$. The presence of obstruction was not affected by sex assignment. Use of non-birth sex moved some FVC and $\mathrm{FEV}_{1}$ data above the lower limit of normal and improved severity classification in others. For born female subjects, percent of predicted for FVC and $\mathrm{FEV}_{1}$ were significantly lower when the male sex was used: $102 \%$ versus $87.5 \%$ and $81.5 \%$ versus $70.5 \%$, respectively $(P<.001)$. FEV $/$ FVC $\mathrm{Z}$ score was $\mathbf{- 2 . 1 7}$ for female sex and $\mathbf{- 2 . 1 2}$ for male sex $(P<.001)$. Six born female subjects had their $\mathrm{FEV}_{1} / \mathrm{FVC}$ normalized when male sex was used. Use of non-birth sex moved some FVC and $\mathrm{FEV}_{1}$ data below the lower limit of normal and worsened severity classification in others. In total, using the non-birth sex affected spirometry interpretation in $45 \%$ of born male subjects and $70 \%$ of born female subjects. CONCLUSIONS: In transgender subjects with air-flow obstruction, using non-birth sex to calculate predicted spirometry values may have a significant impact on test interpretation and place these patients at risk for misdiagnosis and inappropriate treatment. Key words: spirometry; chronic obstructive pulmonary disease; airway obstruction; respiratory function testing; transgender persons. [Respir Care 2018;63(2):215-218. (C) 2018 Daedalus Enterprises]
\end{abstract}

\section{Introduction}

Sex is an important determinant of lung capacity and function. ${ }^{1}$ Clinicians may not be prepared to decide which sex should be used to calculate predicted spirometry values in transgender patients: birth sex or non-birth sex.

\footnotetext{
Mr Haynes is affiliated with the Pulmonary Function Laboratory, St Joseph Hospital, Nashua, New Hampshire. Mr Stumbo is affiliated with the Departments of Pulmonary, Sleep, and Critical Care Medicine, Kaiser Permanente, Tacoma, Washington.
}

Mr Stumbo presented a version of this work at the AARC Congress 2016, held October 15-18, 2016, in San Antonio, Texas.

Mr Haynes has disclosed a relationship with Morgan Scientific. Mr Stumbo has disclosed relationships with Apex Pulmonary Solutions and Thayer Medical.
Although it may seem obvious to some that birth sex should be used for the calculation of predicted spirometry values, there are differences of opinion among clinicians on which sex should be used, and patients may feel that their right to gender identity has been violated if birth sex is used. In addition, electronic medical record systems may populate the pulmonary function patient demographics with the nonbirth sex, conceivably without the technologist or interpreting physician realizing it. This study examined the

\footnotetext{
Correspondence: Jeffrey M Haynes RRT RPFT FAARC, Pulmonary Function Laboratory, St Joseph Hospital, 172 Kinsley Street, Nashua, NH 03060. E-mail: jhaynes@sjhnh.org.
}

DOI: $10.4187 /$ respcare. 05586 
impact of using non-birth sex on the interpretation of spirometry data in subjects with air-flow obstruction.

\section{Methods}

This study was a retrospective analysis of anonymous, publicly available spirometry data (LungXplorer, www.spirxpert.com, Accessed October 19, 2017). Eighty adult male subjects and 80 adult female subjects with airflow obstruction were chosen from the database via random sampling with representation from different decades

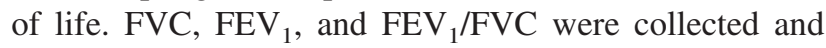
analyzed using the Global Lung Function Initiative reference equations. ${ }^{2}$ Each subject had their spirometry data analyzed using predicted values for the male and female sex. Because the LungXplorer database does not specify subject race, all data were analyzed using the white equations. Differences in percent of predicted, $\mathrm{Z}$ scores, classification of disease severity, ${ }^{3}$ and the incidence of a value migrating above or below the lower limit of normal (lower limit of normal $\mathrm{Z}$ score $=-1.64$ ) between the sex assignments were examined.

\section{Statistical Analysis}

Statistical computations were made using commercially available software (Prism 4, GraphPad Software, La Jolla, California). Median differences in paired data were examined with the Wilcoxon signed-rank test. A 2-tailed $P<.05$ was considered significant.

\section{Results}

Baseline demographic data are listed in Table 1. For born male subjects, the median (interquartile range [IQR]) percent-of-predicted values for FVC and $\mathrm{FEV}_{1}$ were significantly higher when the female sex was used: $100.5 \%$ $(90.5-110.5 \%)$ versus $118.5 \%(105.5-131.5 \%)$ and $78 \%$ $(60-84 \%)$ versus $91.5 \% \quad(70.5 \%-99 \%)$, respectively $\left(P<.001\right.$ for both). The median (IQR) $\mathrm{FEV}_{1} / \mathrm{FVC} \mathrm{Z}$ score was $-2.53(-3.44$ to -2.19$)$ for male sex and -2.65 $(-3.43$ to -2.24$)$ for female sex $(P=.004)$. In all comparisons, the presence of obstruction was not affected by sex assignment (see Fig. 1). Use of non-birth sex moved some FVC and $\mathrm{FEV}_{1}$ data above the lower limit of normal: FVC 2 of $80(2.5 \%)$ and $\mathrm{FEV}_{1} 17$ of $80(21.2 \%)$. In the data that remained below the lower limit of normal, severity classification was improved (less severe) in some subjects: FVC 5 of $78(6.4 \%)$ and FEV $_{1} 23$ of $63(36.5 \%){ }^{3}$ For born female subjects, the median (IQR) percent of predicted for $\mathrm{FVC}$ and $\mathrm{FEV}_{1}$ were significantly lower when the male sex was used: $102 \%(90-113.5 \%)$ versus $87.5 \%$ (77-97.5\%) and $81.5 \%(68-93.5 \%)$ versus $70.5 \%(58.5-$ $81.5 \%)$, respectively $(P<.001$ for both). The median

\section{QUICK LOOK}

\section{Current knowledge}

Sex is an important determinant of lung capacity and function. Expected spirometry values are affected by many factors, including age, height, race, and sex.

\section{What this paper contributes to our knowledge}

In transgender subjects with air-flow obstruction, using non-birth sex to calculate predicted spirometry values may have a significant impact on test interpretation and place these patients at risk for misdiagnosis and inappropriate treatment.

(IQR) $\mathrm{FEV}_{1} / \mathrm{FVC} \mathrm{Z}$ score was $-2.17(-2.54$ to -1.95$)$ for female sex and $-2.12(-2.48$ to -1.86$)$ for male sex $(P<.001)$. Six born female subjects had their $\mathrm{FEV}_{1} / \mathrm{FVC}$ normalized when the male sex was used (see Fig. 2). Severity classification (without change in normality classification) was worsened in some subjects: FVC 9 of 67 (13.4\%) and $\mathrm{FEV}_{1} 29$ of $59(49.1 \%)$. $^{3}$ In total, using the non-birth sex affected spirometry interpretation in $45 \%$ of born male subjects and $70 \%$ of born female subjects.

\section{Discussion}

In this study, using the non-birth sex to calculate predicted spirometry values frequently affected test interpretation. In subjects born males, both $\mathrm{FVC}$ and $\mathrm{FEV}_{1}$ percent of predicted were significantly higher using the non-birth sex; however, $\mathrm{FEV}_{1}$ interpretation more frequently normalized or became less severe. The median $\mathrm{FEV}_{1} / \mathrm{FVC} \mathrm{Z}$ score was more negative when using the non-birth sex. In all comparisons, the presence of obstruction $\left(\mathrm{FEV}_{1} / \mathrm{FVC} \mathrm{Z}\right.$ score $<-1.64)$ was not affected by sex assignment. In born female subjects, both $\mathrm{FVC}$ and $\mathrm{FEV}_{1}$ percent of predicted were significantly lower using the non-birth sex. $\mathrm{FEV}_{1}$ interpretation was also more frequently affected than FVC. The median $\mathrm{FEV}_{1} / \mathrm{FVC} \mathrm{Z}$ score was less negative when using the non-birth sex. In contrast to born male subjects, 6 born female subjects had a normalized $\mathrm{FEV}_{1} / \mathrm{FVC}(\mathrm{Z}$ score $\geq-1.64)$, falsely indicating a normal test result.

Expected spirometry values are affected by many factors, including age, height, race, and sex. ${ }^{2}$ Accordingly, pulmonary function technologists need to be diligent when entering patient demographics to calculate predicted spirometry values. Height should be measured in stocking feet, date of birth must be verified, race should be discussed with the patient, and birth sex (and not gender identity) should be used to calculate predicted spirometry 
Table 1. Baseline Subject Demographics

\begin{tabular}{|c|c|c|}
\hline Characteristics & Born Male $(n=80)$ & Born Female $(n=80)$ \\
\hline Age, median (IQR) y, range & $44(31.5-59.5), 17-82$ & 44.5 (31.5-57), 19-80 \\
\hline \multicolumn{3}{|l|}{ Pulmonary function } \\
\hline FVC, median (IQR) \% predicted, range & 100.5 (90.5-110.5), 30-136 & 102 (90-113.5), 50-137 \\
\hline $\mathrm{Z}$ score, median (IQR), range & 0.03 ( -0.76 to 0.79$),-4.50$ to 2.85 & $0.17(-0.75$ to 1.06$),-4.20$ to 2.94 \\
\hline $\mathrm{FEV}_{1}$, median (IQR) \% predicted, range & 78 (60-84), 19-111 & $81.5(68-93.5), 29-113$ \\
\hline $\mathrm{Z}$ score, median (IQR), range & $-1.72(-2.84$ to 1.14$),-4.59$ to 1.69 & $-1.38(-2.23$ to -0.51$),-4.59$ to 1.12 \\
\hline \multicolumn{3}{|l|}{$\mathrm{FEV}_{1} / \mathrm{FVC}$} \\
\hline $\mathrm{Z}$ score, range & $-2.53(-3.44$ to -2.19$),-4.91$ to -1.81 & $-2.17(-2.54$ to -1.95$),-4.33$ to -1.67 \\
\hline
\end{tabular}

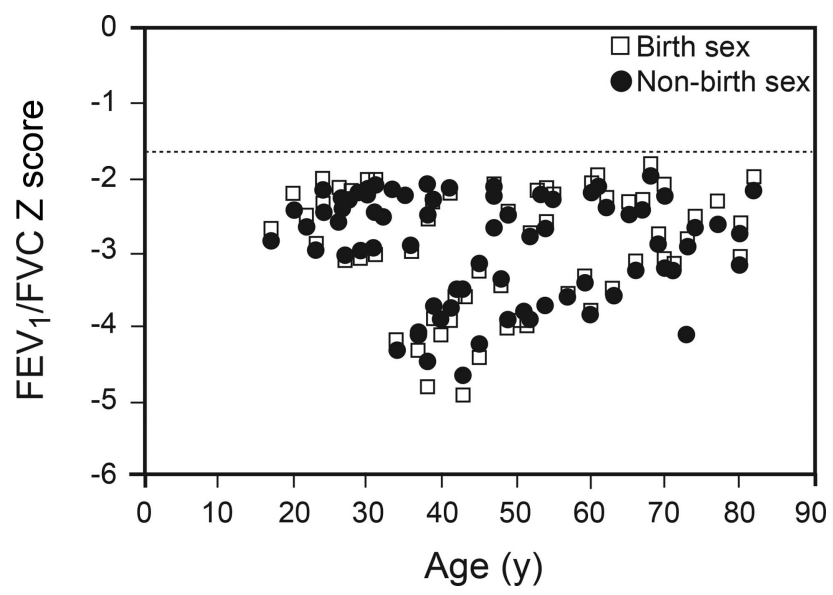

Fig. 1. FEV1/FVC Z scores calculated using birth and non-birth sex in born male subjects. Dotted line: lower limit of normal.

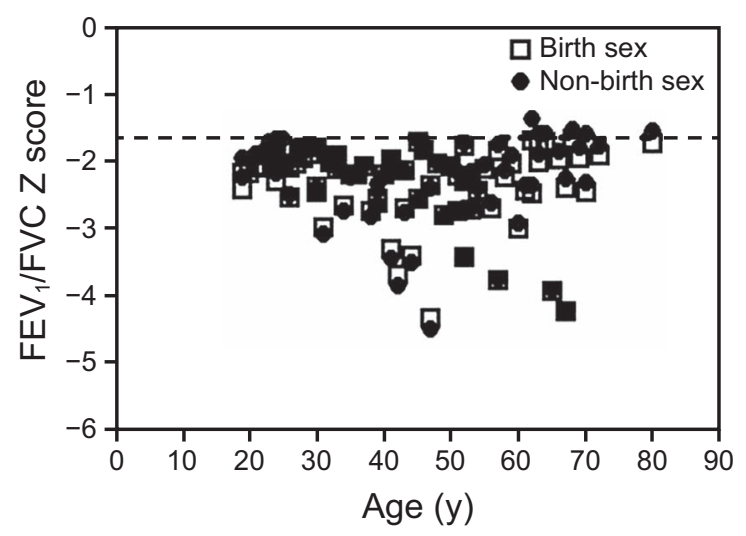

Fig. 2. FEV1/FVC Z scores using birth and non-birth sex in born female subjects.

values. For the purposes of pulmonary function testing, a distinction must be made between birth sex and gender identity. The Merriam-Webster dictionary defines gender identity as "a person's internal sense of being male, female, some combination of male and female, or neither male or female" (http://www.merriam-webster.com, Accessed February 7, 2017). This study provides scientific justification for using birth sex to calculate spirometry predicted values in transgender individuals. Although we recommend that predicted spirometry values be calculated using birth sex, the demographic profile recorded in pulmonary function systems and electronic medical records should also include gender identity. Including both birth sex and gender identity in electronic medical records, the so-called 2-step approach, has been recommended by LGBT advocates. ${ }^{4,5}$ This approach would both preserve the accuracy of reported pulmonary function values and protect a patient's right to determine their gender identity.

Unfortunately, with numerous spirometer manufacturers and a vast number of devices in use, achieving universal adoption of transgender-appropriate software could take years if not decades. A novel, interim solution is to create transgender predicted equations. The transgender predicted equation would simply be an existing equation calculated with birth sex but linked to the patient's gender identity. For example, for a transwoman, the technologist would enter female into the patient demographics but would select the transgender predicted equation calculated with birth sex. We believe that the transgender predicted equation strategy would accomplish the same goals as the 2-step approach. In addition, the transgender predicted equation strategy would obviate any need to make report modifications before transmission into an electronic medical record (eg, technologist notes regarding the patient's birth sex and gender identity). In the longer term, a universally adopted solution to this problem must be achieved. Failure to recognize a patient's right to determine their gender identity can cause significant emotional and psychological harm and may discourage patients from accessing needed medical care. 


\section{Non-Birth SeX and Spirometry Data Interpretation}

Our study has several limitations. We only examined the impact of using the non-birth sex in subjects with air-flow obstruction. Studies on the impact of using non-birth sex in those with normal and restrictive lung disease should be conducted. In addition, this study only assessed the impact of using non-birth sex on spirometry interpretation according to one reference equation using white race. ${ }^{2}$ Whereas some of the raw data were presumably collected from non-white subjects, these FVC and $\mathrm{FEV}_{1}$ values could be collected from subjects of any ethnic group with the same age and height. Moreover, we defined air-flow obstruction as an $\mathrm{FEV}_{1} / \mathrm{FVC}$ $\mathrm{Z}$ score $<-1.64$, which applies to all races. ${ }^{6}$ Last, we only examined adult subjects who presumably did not receive gender-guided hormone therapy. Whereas it is known that lung development is impacted by hormones starting before and during the neonatal period, ${ }^{7}$ it is unclear whether hormone augmentation during adolescence can impact adult lung capacity and function. The size of the transgender population is small $(0.39 \%)^{8}$; however, pulmonary function technologists can expect to test more transgender individuals as societal attitudes toward the transgender population become more supportive.

\section{Conclusions}

In transgender subjects with air-flow obstruction, using non-birth sex to calculate predicted spirometry values may have a significant impact on test interpretation and place these patients at risk for misdiagnosis and inappropriate treatment. Spirometry testing can be conducted with physiologic accuracy without violating a patient's right to gender identity.

\section{REFERENCES}

1. Harms CA. Does gender affect pulmonary function and exercise capacity? Respir Physiol Neurobiol 2006;151(2):124-131.

2. Quanjer PH, Stanojevic S, Cole TJ, Baur X, Hall GL, Culver BH, et al. Multi-ethnic reference values for spirometry for the 3-95-yr age range: the global lung function 2012 equations. Eur Respir J 2012; 40(6):1324-1343.

3. Pellegrino R, Viegi G, Brusasco V, Crapo RO, Burgos F, Casaburi R, et al. Interpretative strategies for lung function tests. Eur Respir J 2005;26(5):948-968.

4. Deutsch MB, Buchholz D. Electronic health records and transgender patients: practical recommendations for the collection of gender identity data. J Gen Intern Med 2015;30(6):843-847.

5. Cahill S, Makadon H. Sexual orientation and gender identity data collection in clinical settings and in electronic health records: a key to ending LGBT health disparities. LGBT Health 2014;1(1):34-41.

6. Stanojevic S, Wade A, Stocks J. Reference values for lung function: past, present and future. Eur Respir J 2010;36(1):12-19.

7. Carey MA, Card JW, Voltz JW, Arbes SJ, Jr, Germolec DR, Korach KS, and Zeldin DC. It's all about sex: gender, lung development and lung disease. Trends Endocrinol Metab 2007;18(8):308-313.

8. Meerwijk EL, Sevelius JM. Transgender population size in the United States: a meta-regression of population-based probability samples. Am J Public Health 2017;107(2):e1-e8. 